\title{
LA WEBQUEST COMO ESTRATEGIA METODOLÓGICA EN LA ENSEÑANZA UNIVERSITARIA DE LA ASIGNATURA DE CONTABILIDAD DE EMPRESAS TURÍSTICAS
}

\author{
THE WEBQUEST AS A LEARNING MODEL AT THE UNIVERSITY. AN \\ APPLICATION TO ACCOUNTING LEARNERS OF TOURISM DEGREE
}

\author{
Paloma del Campo Moreno; mcampo@cee.uned.es \\ Laura Parte Esteban; Iparte@cee.uned.es \\ Universidad Nacional de Educación a Distancia (UNED)
}

\begin{abstract}
RESUMEN
En este artículo se describe la aplicación de una estrategia de aprendizaje por descubrimiento utilizando la técnica del webquest a la asignatura Contabilidad de Empresas Turísticas de la Diplomatura de Turismo de la UNED. Su realización se ha revelado como una actividad estimulante para los estudiantes porque les hace ser protagonistas del proceso de enseñanza-aprendizaje; el profesor toma el papel de "guía", construyendo el andamiaje que soporta toda la actividad y que orienta al alumno para que autónomamente pueda llevar a cabo su trabajo y adquiera las habilidades y destrezas específicas de la materia.
\end{abstract}

PALABRAS CLAVE: Docencia universitaria, Nuevas Tecnologías, Webquest,

\section{ABSTRACT}

This paper describes a learning experience based on webquest driven by student of the Spanish National University for Distance Education (UNED). In particular, we develop the experience in the Tourism Degree with accounting students. As a pedagogic tool, the webquest is an inquiry-oriented activity that presents important educational possibilities. The learners face up to a stimulating activity where they are the main protagonist. The teachers provide the orientations to develop the students' attitudes to achieve the knowledge driven by self-learning process.

KEY WORDS: Higher Education, New Technologies, Webquest, 


\section{INTRODUCCIÓN}

El nuevo escenario que se presenta, derivado de la entrada en vigor de las nuevas titulaciones adaptadas a las directrices del Espacio Europeo de Educación Superior (EEES) exige poner en práctica nuevas formas de enseñar que permitan al estudiante desarrollar las habilidades, competencias y destrezas requeridas en el perfil formativo del título. La puesta en práctica de estrategias de aprendizaje adaptadas a este nuevo entorno educativo permite adquirir experiencia tanto a los profesores, sobre la forma de enseñar su materia, como a los estudiantes, sobre la forma de aprender y aplicar los conocimientos adquiridos. Factores como la estrategia metodológica a emplear para formar en competencias, la valoración del tiempo y esfuerzo para su diseño e implantación, los pros y contras de su aplicación, el diseño de la evaluación, etc., son algunos de los interrogantes que se abren paso y para los que los docentes tienen que buscar respuesta.

En este artículo se describe la puesta en práctica de una experiencia encaminada a ensayar nuevas técnicas de enseñanza-aprendizaje dentro del marco del EEES y el análisis posterior de su eficacia. Concretamente, se centra en el análisis de la webquest como estrategia metodológica en la asignatura Contabilidad de Empresas Turísticas que se imparte en el segundo curso de la Diplomatura de Turismo en la UNED. Se trata de una asignatura de contenido abstracto y con una fuerte carga de trabajo práctico, lo que hace que su asimilación tenga un periodo de maduración largo. Hay que considerar además que, cuando los estudiantes se enfrentan a esta asignatura, cuentan con unos conocimientos previos insuficientes 0 , en muchos casos, poco consolidados porque previamente tan sólo han cursado 5 créditos de Introducción a la Contabilidad. Todas estas características hacen que la asignatura sea percibida por ellos como "difícil". Con frecuencia, los estudiantes realizan los ejercicios que se recogen tradicionalmente en los manuales de Contabilidad de Costes sin percibir la evidente y fundamental aplicación práctica de la asignatura en el ámbito empresarial.

Todos estos factores nos animaron a diseñar una webquest con la pretensión de que los alumnos mejoraran su predisposición y motivación hacia la asignatura y percibieran la utilidad de lo que estudian. Nuestra experiencia se suma a los trabajos previos realizados en el ámbito de la enseñanza universitaria y aporta evidencia adicional sobre la eficacia y utilidad de esta técnica aplicada a la materia Contabilidad.

A continuación se hace una reflexión sobre la idoneidad de esta estrategia metodológica en el marco del EEES, se explica su aplicación a la asignatura Contabilidad de Empresas Turísticas y se analizan los resultados obtenidos. Al final del artículo se exponen una serie de reflexiones derivadas de la experiencia. 


\section{LA IDONEIDAD DE LA WEBQUEST EN EL EEES}

La implantación de los créditos europeos va a suponer un cambio significativo en el papel del profesor en los procesos a aprendizaje. Tal como señala Palacios (2009, p.236) esos cambios incluyen varios aspectos como son la metodología docente, el rol del profesor y el fomento de nuevos materiales y nuevos sistemas de evaluación.

En este modelo, el trabajo del alumno pasa a primer término, lo que supone que se dé más importancia a los procesos de aprendizaje que a los procesos de enseñanza y que se prime el "aprender a aprender" por encima de la memorización de contenidos. El profesor debe desempeñar el rol de tutor, guía o facilitador del proceso de aprendizaje de los estudiantes que ahora serán más emprendedores y autónomos.

Las estrategias formativas que se pongan en marcha deben ser variadas para permitir al alumno no sólo adquirir competencias específicas de la materia que estudia sino también desarrollar destrezas y habilidades que tienen que ver con colaborar y comunicarse, interactuar y aprender con sus compañeros, el fomento de la creatividad, el análisis crítico, la selección e interpretación de datos, el trabajo autónomo, etc.

El concepto de webquest fue propuesto por Dodge para ayudar a identificar el nuevo tipo de actividades que se estaban llevando a cabo para el uso de internet en el ámbito educativo (Barba, 2004). La webquest se trata de una actividad orientada para la investigación en la que se trabaja con información que proviene de internet y con la que se propone una tarea factible y atractiva para los estudiantes y un proceso para realizarla. Se trata, tal como explica Adell (2004, p. 2) de que los alumnos manejen información, la analicen, sinteticen, comprendan, juzguen, valoren, creen nueva información, etc.

Es, por sus características, una metodología que cumple con los requisitos del nuevo modelo universitario nacido de Bolonia al ser una estrategia de aprendizaje activo. Ya existen numerosas experiencias que así lo avalan (Bernabé y Adell, 2006; Bernabé, 2007; Lara, 2007; Rodera 2008; Degrossi, 2009; Palacios, 2009, entre otras). El profesor propone una actividad motivadora, cuyo contenido tiene una conexión con la práctica profesional y trata aspectos interesantes para la formación de los estudiantes o para su experiencia personal (March, 2000), pero son los estudiantes los que asumen la mayor parte del trabajo y deben llevar la iniciativa en la elaboración del mismo.

Al adoptarla en nuestra labor docente nos propusimos como objetivos:

- Profundizar en los fundamentos pedagógicos de la webquest.

- Adaptar el modelo de la webquest al ámbito universitario.

- Promover su uso como recurso didáctico para llevar a término procesos de enseñanza y aprendizaje utilizando internet y otras TIC. 
- Experimentar cómo trabajar en la enseñanza a distancia de manera colaborativa utilizando la webquest.

- Evaluar el proceso y los resultados finales de la experiencia frente a la metodología tradicional que veníamos aplicando, detectando las ventajas e inconvenientes de su aplicación.

- Conocer y compartir las experiencias en el uso de las webquests que se desarrollen en la universidad a distancia y en la universidad presencial.

\section{APLICACIÓN DE LA WEBQUEST EN LA ASIGNATURA CONTABILIDAD DE EMPRESAS TURÍSTICAS}

En el curso 2008/2009 el equipo docente de esta asignatura decidió inscribirse en un proyecto de investigación en innovación docente financiado por la UNED participando así en una investigación que trata de dar respuesta a los requerimientos dispuestos en el marco del EEES. Particularmente, se trata de una investigación que se sitúa en la línea de la aplicación de nuevas estrategias metodológicas para el EEES y que se articula como un proyecto piloto cuya participación, para los estudiantes, es de carácter voluntario.

Hasta ese momento la asignatura se había impartido siguiendo la metodología propia de la enseñanza a distancia, basada fundamentalmente en proporcionar al estudiante un material básico escrito que desarrolla todo el programa, tanto en su vertiente conceptual o teórica como en la aplicada y que se ha redactado ad hoc para permitir el trabajo autónomo, y en el uso del curso virtual para la difusión de material complementario y la comunicación entre profesores y estudiantes.

Por su parte, la nueva experiencia metodológica se ha basado, en:

- El uso de la plataforma WebCt para:

- Difundir el calendario detallado con las actividades a realizar y las fechas de entrega de las mismas.

- Colocar el material necesario para realizar las tareas establecidas.

- Llevar a cabo una comunicación fluida estudiante-profesor y estudiantes entre sí.

- Supervisar el trabajo realizado por los participantes.

- $\quad$ La realización de una webquest.

- $\quad$ El diseño y distribución de un cuestionario de satisfacción dónde se recogen las opiniones de los estudiantes sobre el trabajo desarrollado. La información proporcionada sirve para evaluar la experiencia y como retroalimentación para el desarrollo de la actividad en cursos futuros. 
La webquest constituye el eje central de esta experiencia, y por sus características, es una metodología que está en consonancia con los requerimientos del EEES, ya que exige que el estudiante represente un papel de protagonista en el proceso de su formación y que el profesor pase a un segundo plano, representando el papel de orientador y guía en ese proceso. Así, la iniciativa pasa de profesor al estudiante y del "enseñar" al "aprender".

Los objetivos pretendidos con la aplicación de la webquest fueron:

- Despertar en el estudiante el interés por la asignatura.

- Conectar los contenidos de la asignatura con sus aplicaciones prácticas.

- Que el estudiante establezca la relación global entre todos los temas del programa.

- Estructurar el conocimiento del estudiante mediante el análisis, la selección, y la interpretación de información relacionada con una situación "real" centrada en los contenidos de la asignatura.

- Descubrir la utilidad de lo que estudia.

Y además:

- Promover la capacidad creativa.

- Promover la capacidad crítica.

- Capacidad de análisis y síntesis.

- Mejorar las destrezas en el uso de la informática.

- Promover el trabajo autónomo y autorregulado.

- Capacidad de organización y planificación.

- Capacidad de generar ideas innovadoras.

Cabe señalar que no planteamos un webquest en su estado puro, dado que el trabajo se llevó a cabo de forma individual para salvar, en esta ocasión, las dificultades que el trabajo en equipo plantea en la enseñanza a distancia y tampoco facilitamos a los estudiantes todas las fuentes de información que tenían que utilizar sino tan sólo algunas orientaciones sobre la localización de las mismas, de modo que introdujimos así una experiencia de investigación que enriquecía el trabajo y que creemos, es propia, del ámbito universitario. Por lo demás, nuestra webquest se diseñó siguiendo un planteamiento tradicional que consta de 5 fases: Introducción, Tarea, Proceso, Recursos, Evaluación y Conclusiones. A continuación explicamos el contenido de cada etapa. 


\section{1. Introducción}

En esta etapa se explicaron los objetivos que se pretendían conseguir con el trabajo y se plantearon las preguntas que los alumnos debían contestar. Si hemos de destacar algún aspecto de esta fase es el papel motivador que representa. Se trata de despertar el interés de los estudiantes y las ganas de implicarse en la tarea. En esta etapa se asigna a cada estudiante el rol que debe representar y se delimita el escenario en el que se debe mover.

En nuestro caso se les pidió que representaran el papel de profesor para que explicaran a sus compañeros la utilidad que tiene la asignatura y la conexión de sus contenidos con el mundo de la empresa. Concretamente se les decía: "el objetivo de esta webquest es conseguir que seas capaz de desarrollar el organigrama técnico de la empresa, es decir, la representación gráfica y esquematizada del proceso productivo de la empresa, el cual, a su vez, te permitirá localizar, clasificar, periodificar e imputar los costes a los productos, para determinar con el menor sesgo posible su coste unitario de producción".

Con los argumentos ofrecidos en esta parte de la webquest se busca que los estudiantes perciban que es el proceso técnico-económico de la empresa el que condiciona el diseño de la Contabilidad de Costes y hace que sea distinto en una empresa que en otra. Además, se pretende que observen que el modelo base que se estudia en la asignatura sirve para captar el movimiento interno de valores que se produce en cualquier empresa y que tiene, por tanto, una evidente aplicación práctica.

\section{2. La tarea}

Esta etapa contiene los objetivos que los estudiantes deben alcanzar, esto es, las tareas a realizar y los métodos más adecuados para llevarla a cabo. Es la descripción de lo que el estudiante debe obtener al concluir todas las actividades que se proponen en la webquest. Es importante presentar la tarea de la manera más clara y precisa posible (puede consultarse la taxonomía de tareas que propone Dodge, 1999).

En nuestra webquest se pidió que elaboraran un documento word, página web, powerpoint, es decir, que llevaran a cabo una presentación multimedia con la descripción de un proceso técnico-productivo y la representación gráfica del mismo. Así, además de adquirir las competencias específicas de la materia, entrenarían sus destrezas en el uso de herramientas informáticas básicas. También pensamos que sería pertinente que practicaran la habilidad de la comunicación oral, de modo que solicitamos a los estudiantes que acompañaran su presentación con una grabación de audio para resumir los puntos más importantes de su trabajo estableciéndose así una semejanza con la defensa oral que se realiza habitualmente en la enseñanza presencial. 
Los participantes contaron con una planificación detallada de los diferentes puntos que debían desarrollar. Para ello los profesores elaboramos una serie de preguntas a las que debían dar respuesta de forma secuencial; constituían el guión del informe. Estas preguntas orientan sobre el contenido que debe ser abordado, ahorman la estructura que debe tener el producto final que se les pide e hilvanan los contenidos del mismo. Una formulación clara, adecuada y detallada propicia el que los estudiantes puedan trabajar con mayor autonomía (véase el cuadro 1).

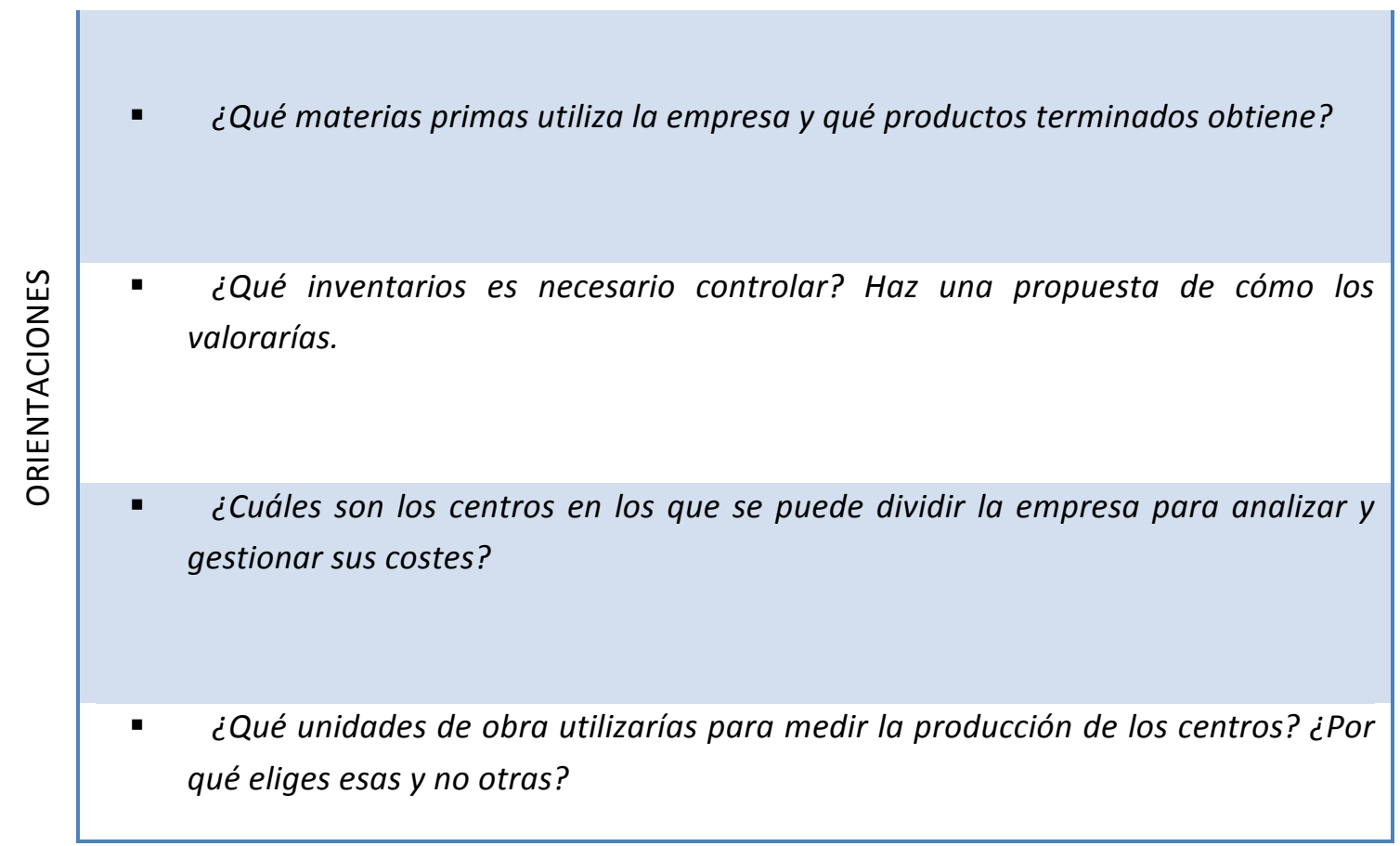

Cuadro 1. PASO 2: LA TAREA 


\section{3. El proceso}

En esta etapa se detallan los pasos a seguir para conseguir el producto final. Se explica a los alumnos cuándo, cómo y qué deben hacer. Se trata de repartir las tareas de acuerdo con un horizonte temporal que marque los plazos a cumplir. Aquí igualmente, conviene ofrecer la información con un gran nivel de detalle. En nuestra webquest se indicaron los siguientes puntos (véase el cuadro 2)

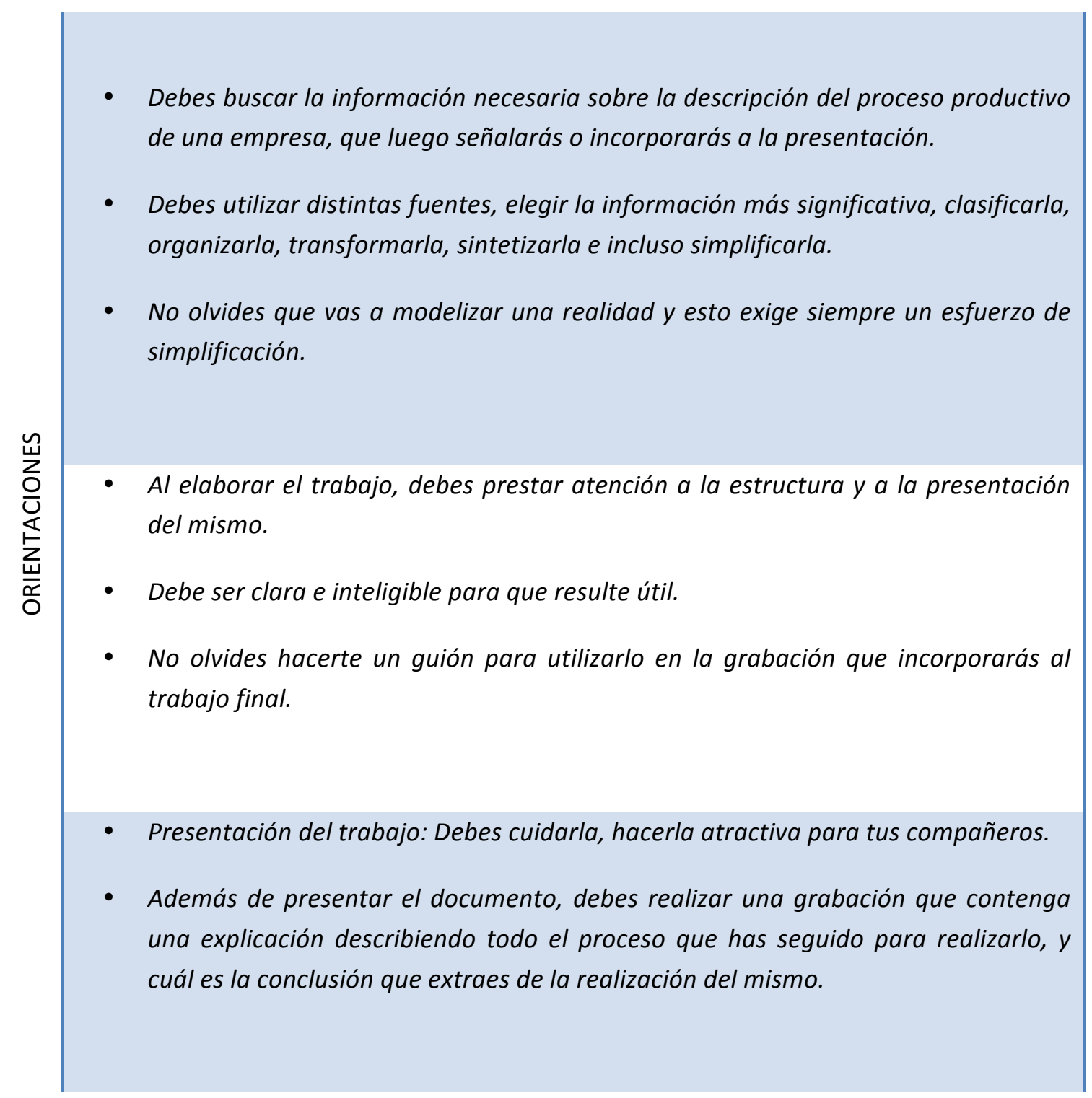

Cuadro 2. PASO 3: EL PROCESO

\section{4. Los recursos}

En este apartado procede citar las fuentes a las que deben remitirse los estudiantes para recopilar el material que les permitirá desarrollar el producto. Esta etapa contiene enlaces a sitios web donde se puede encontrar la información requerida para la realización de las distintas tareas y que pueden tener diversos formatos, tales como gráficos interactivos, 
vídeos, o documentos html. Además, pueden ser complementados con materiales específicos elaborados por el equipo docente.

En un primer paso, el profesor debe realizar la primera criba de las fuentes de información que se proporcionan. Como segundo paso, es necesario que los estudiantes analicen la información puesta a su disposición. Son ellos los que deben comprender, valorar, y decidir qué datos van a manejar. Se trata de que construyan conocimiento nuevo fruto del grado de asimilación de la información seleccionada y del conocimiento de la materia que cada uno haya alcanzado.

En nuestra webquest se facilitaron direcciones web para consultar procesos productivos de empresas que desarrollan actividades muy variadas, que van desde la fabricación de latas hasta la elaboración de queso, por ejemplo.

\section{5. La evaluación}

La evaluación cumple la función de informar al estudiante acerca de cómo se va a valorar su trabajo. Saber de antemano o a priori cómo se va a medir el rendimiento del estudiante le orienta, le motiva y también le muestra lo que se espera de él. La técnica de valoración más utilizada aplicada a este tipo de actividades es la rúbrica (Dodge, 2001a, 2001b; Conde y Pozuelo, 2007). La rúbrica es una plantilla que contiene y concreta los criterios de evaluación. En nuestro caso se elaboró la siguiente rúbrica (véase el cuadro 3).

\begin{tabular}{|c|l|l|l|l|}
\hline CONCEPTOS & \multicolumn{1}{|c|}{ NO APTO } & \multicolumn{1}{|c|}{ BIEN } & \multicolumn{1}{|c|}{ NOTABLE } & \multicolumn{1}{|c|}{ SOBRESALIENTE } \\
\hline \multirow{5}{*}{ Contenido } & $\begin{array}{l}\text { Trabajo } \\
\text { incompleto. } \\
\text { Sin datos. } \\
\text { Sin análisis } \\
\text { serio. }\end{array}$ & $\begin{array}{l}\text { Puntos } \\
\text { incompletos. } \\
\text { Pocos datos. } \\
\text { Análisis } \\
\text { superficial. } \\
\text { Datos no } \\
\text { contrastados. }\end{array}$ & $\begin{array}{l}\text { Todos los puntos } \\
\text { completos. } \\
\text { Análisis } \\
\text { completo. } \\
\text { Datos } \\
\text { coherentes. }\end{array}$ & $\begin{array}{l}\text { Puntos completos. } \\
\text { Información } \\
\text { relacionada con } \\
\text { varios aspectos de } \\
\text { la temática. } \\
\text { Análisis } \\
\text { exhaustivo. } \\
\text { Datos originales. }\end{array}$ \\
\hline \multirow{5}{*}{$\begin{array}{l}\text { Presentación } \\
\text { No se ofrecen } \\
\text { conclusiones } \\
\text { Se utilizan pocas } \\
\text { herramientas } \\
\text { informáticas o } \\
\text { se utilizan mal } \\
\text { Preguntas sin } \\
\text { contestar }\end{array}$} & $\begin{array}{l}\text { Conclusiones } \\
\text { débiles } \\
\text { Pocas } \\
\text { herramientas } \\
\text { utilizadas } \\
\text { Escaso dominio } \\
\text { del tema } \\
\text { Muchos datos } \\
\text { pero mal } \\
\text { estructurados }\end{array}$ & $\begin{array}{l}\text { Se ofrecen } \\
\text { buenas } \\
\text { conclusiones. } \\
\text { Se utilizan } \\
\text { bastantes } \\
\text { herramientas. } \\
\text { Se realiza una } \\
\text { exposición bien } \\
\text { estructurada }\end{array}$ & $\begin{array}{l}\text { Su seguimiento } \\
\text { resulta } \\
\text { interesante. } \\
\text { Se apoya la } \\
\text { presentación con } \\
\text { herramientas } \\
\text { apropiadas. } \\
\text { Se realiza una } \\
\text { exposición muy } \\
\text { bien estructurada. }\end{array}$ \\
\hline
\end{tabular}




\begin{tabular}{|l|l|l|l|l|}
\hline Gráficos & $\begin{array}{l}\text { Ausencia de } \\
\text { gráficos } y / 0 \\
\text { tablas }\end{array}$ & $\begin{array}{l}\text { Pocos gráficos o } \\
\text { sin conexión con } \\
\text { la exposición }\end{array}$ & $\begin{array}{l}\text { Bastantes } \\
\text { gráficos } \\
\text { relacionados con } \\
\text { la exposición }\end{array}$ & $\begin{array}{l}\text { Gráficos } \\
\text { detallados } \\
\text { originales }\end{array}$ \\
\hline
\end{tabular}

Cuadro 3. Criterios de evaluación

Cabe comentar que el diseño de la webquest contempla en la última etapa las Conclusiones. Se trata de un apartado donde se invita a todos los participantes a reflexionar sobre las tareas realizadas. El feedback que se obtiene del proceso y de la actividad en sí, permite poner en marcha acciones de mejora en futuras prácticas.

En el cuadro 4 se enumeran algunas de las cuestiones planteadas a los participantes de nuestra webquest.

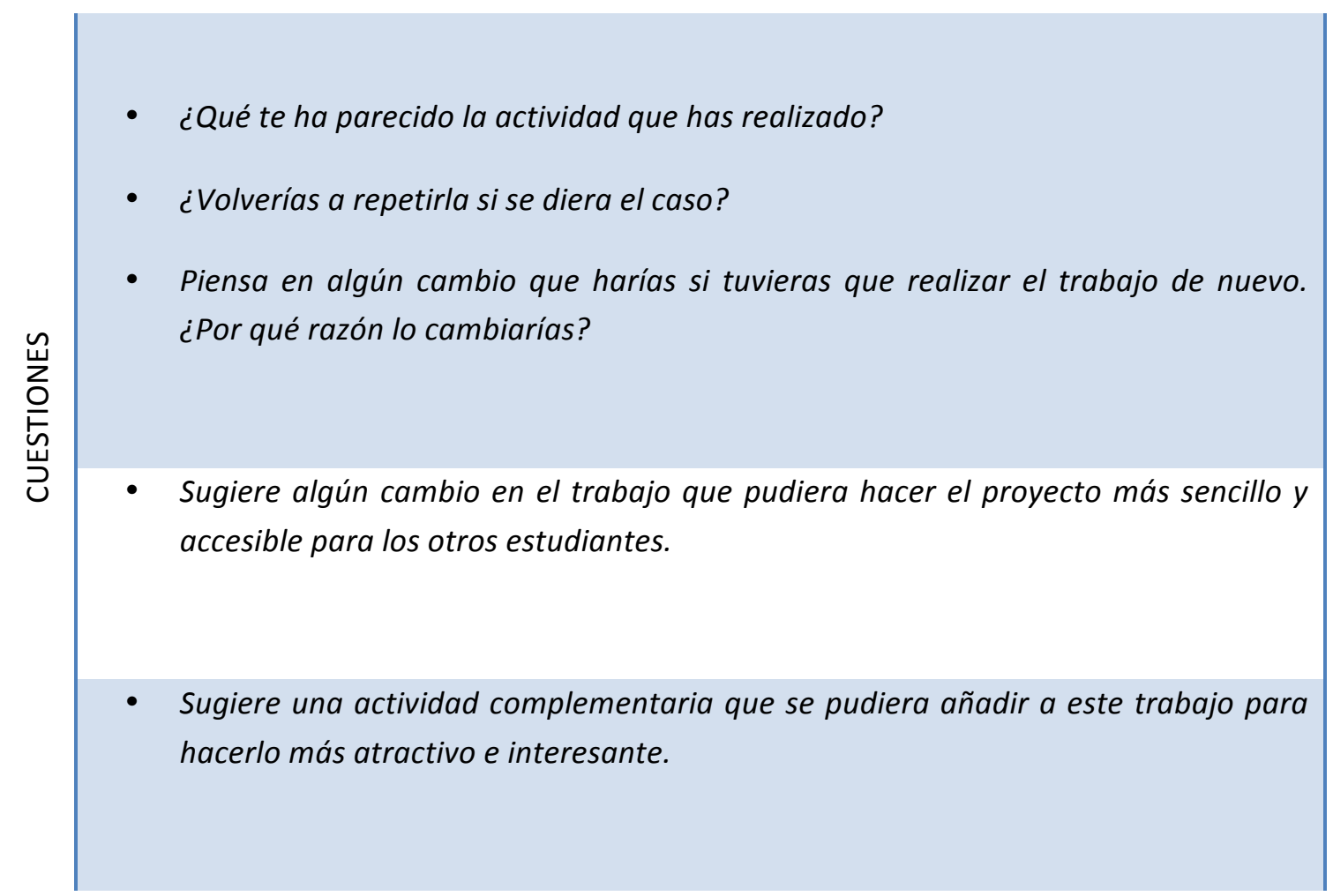

CUadro 4. PASO 5: CONCLUSIONES

\section{ANÁLISIS DE LOS RESULTADOS}

En este apartado se detallan los resultados que se han obtenido con la aplicación de la webquest a la asignatura de Contabilidad de Empresas Turísticas. Durante los dos cursos en los que se ha ofertado este proyecto de innovación docente hemos contado con un total de 20 participantes voluntarios: 6 durante el primer curso y 14 en el segundo.

La estadística descriptiva de la muestra indica que la mayor parte de los participantes son mujeres (75\%). El proyecto de innovación ha tenido cobertura en la Comunidad de Madrid 
(25\%), en otras Comunidades Autónomas (70\%) e incluso fuera de España (5\%). La media de edad de los participantes se ha situado entre los 36 y 45 años (véase gráficos 1).

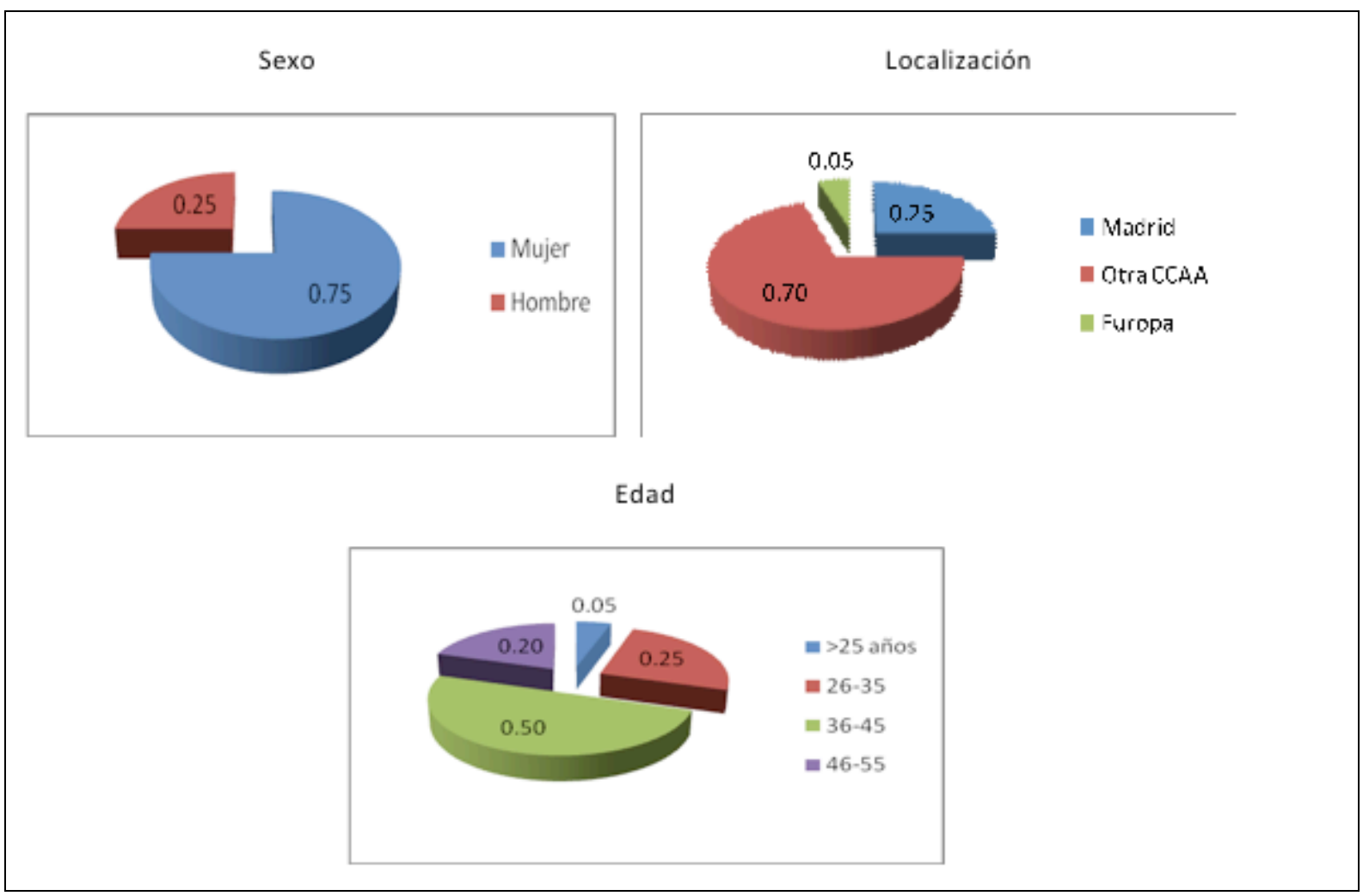

Gráficos 1. Datos de la muestra

La eficacia de la webquest como estrategia metodológica en nuestra asignatura se analizó utilizando la matriz de evaluación presentada anteriormente, las conclusiones expuestas y la encuesta de valoración que los participantes cumplimentaron tras realizar todas las actividades. Las respuestas del cuestionario se plantearon siguiendo una escala Likert con 5 niveles: 1 . Nada de acuerdo; 2. Poco de acuerdo; 3. Acuerdo medio; 4. Bastante de acuerdo; 5. Totalmente de acuerdo.

La valoración de los trabajos de acuerdo con la rúbrica aplicada arroja los siguientes porcentajes: un $80 \%$ de los trabajos presentados superaron con éxito los requisitos mínimos de contenido exigidos mientras que un $20 \%$ de los trabajos estaban incompletos o sin datos, y por tanto, no se ajustaron a las exigencias mínimas de contenido. Por el contrario, el $100 \%$ de los trabajos superó los requisitos mínimos de presentación y gráficos.

Por su parte, en las respuestas a la encuesta de valoración se observa que los participantes están de acuerdo o totalmente de acuerdo en que:

- Las instrucciones dadas me han permitido realizar la actividad autónomamente (un $74 \%$ está de acuerdo o totalmente de acuerdo).

- La realización de esta actividad ha mejorado mi capacidad de organización y 
planificación (un 74\% está de acuerdo o totalmente de acuerdo).

- La realización de esta actividad ha mejorado mi habilidad para gestionar la información (un 74\% está de acuerdo o totalmente de acuerdo).

- La realización de esta actividad ha mejorado mi capacidad de aplicar los conocimientos (un 89\% está de acuerdo o totalmente de acuerdo).

- La realización de la actividad me ha ayudado a entender mejor la asignatura (un 81\% está de acuerdo o totalmente de acuerdo).

- La realización de esta actividad me ha ayudado a ver la aplicación práctica de la asignatura (un $89 \%$ está de acuerdo o totalmente de acuerdo).

- La realización del proyecto me ha servido para preparar el examen de la asignatura (un $70 \%$ está de acuerdo o totalmente de acuerdo).

- Realizando el proyecto he aprendido cosas útiles (un $85 \%$ está de acuerdo o totalmente de acuerdo).

Por el contrario los estudiantes manifiestan no estar nada de acuerdo o poco de acuerdo con que:

- La realización de esta actividad ha sido una pérdida de tiempo (un 84\% está nada de acuerdo).

- A pesar de realizar el proyecto, no he sido capaz de entender la asignatura (un $84 \%$ está nada de acuerdo).

Otros aspectos de la encuesta de valoración cumplimentada por los participantes muestran que las actividades se han planificado correctamente, las orientaciones emitidas han sido claras y las ayudas proporcionadas han servido para guiar la elaboración de los distintos trabajos (véase el gráfico 2 para visualizar algunos de los resultados obtenidos).

En suma, los resultados obtenidos en la encuesta de satisfacción indican que la webquest es una estrategia metodológica eficaz en la enseñanza de la asignatura Contabilidad de Empresas Turísticas. La aplicación de la webquest ha permitido al estudiante adquirir las competencias específicas de la materia (objetivo principal de la experiencia realizada) pero además ha permitido desarrollar habilidades relacionadas con el análisis crítico, la selección e interpretación de datos, el trabajo autónomo, comunicación y el fomento de la creatividad, entre otras.

Los resultados encontrados aportan evidencia adicional en una línea de trabajo que actualmente está acaparando gran atención investigadora, la eficacia de nuevas técnicas de enseñanza-aprendizaje dentro del marco del EEES. Hasta el momento los trabajos realizados en el ámbito de la Contabilidad son escasos, por lo que la presente experiencia contribuye al desarrollo de esta línea de investigación. 
Gráfico 2. Resultados elaborados a partir de la encuesta de satisfacción ${ }^{1}$ :

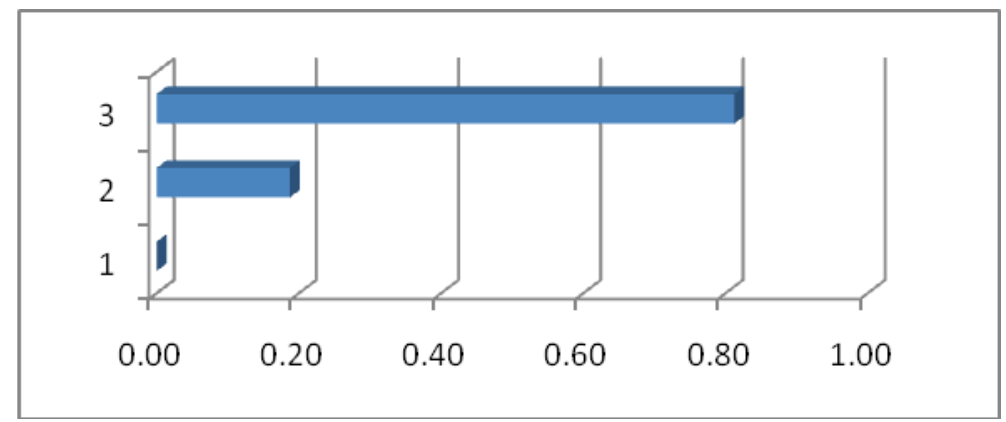

La realización de la actividad me ha ayudado a entender mejor la asignatura

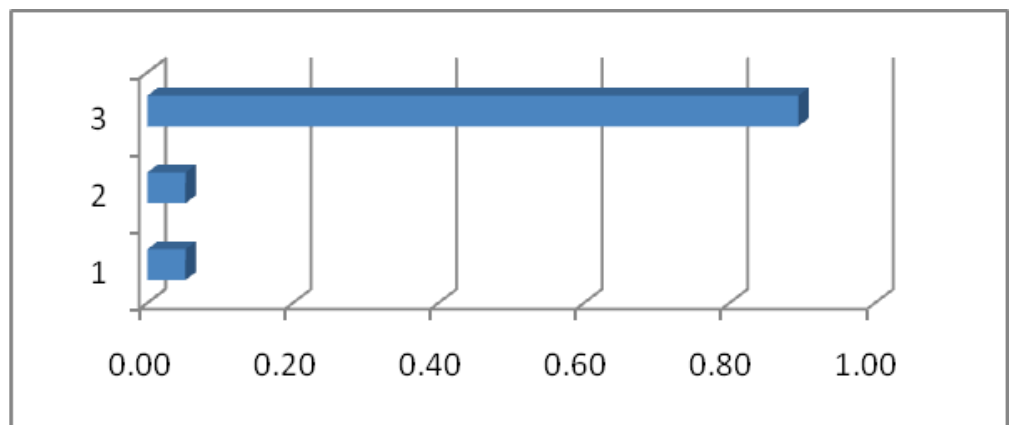

La realización de la actividad me ha ayudado a ver la aplicación práctica de la asignatura

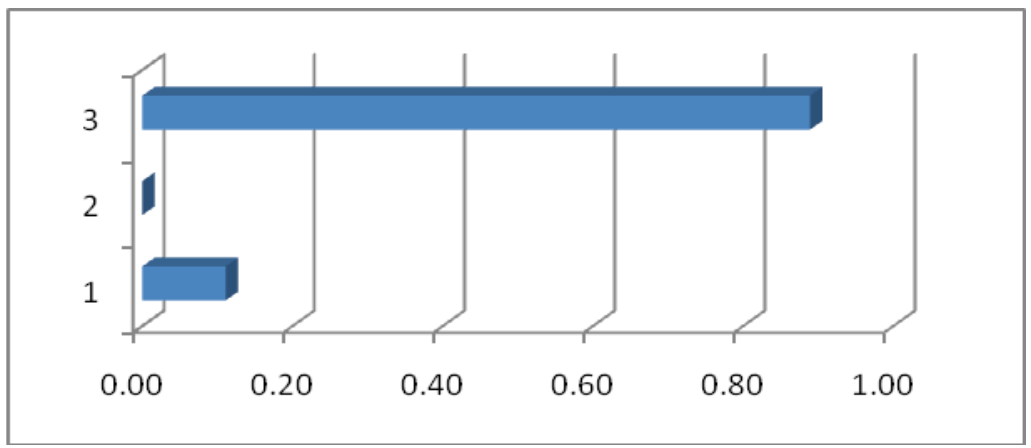

La realización de esta actividad he mejorado mi capacidad de aplicar los conocimientos

\footnotetext{
${ }^{1}$ Siendo 1 Nada de acuerdo o Poco de acuerdo, 2 Acuerdo medio y 3 Bastante de acuerdo o Totalmente de acuerdo.
} 


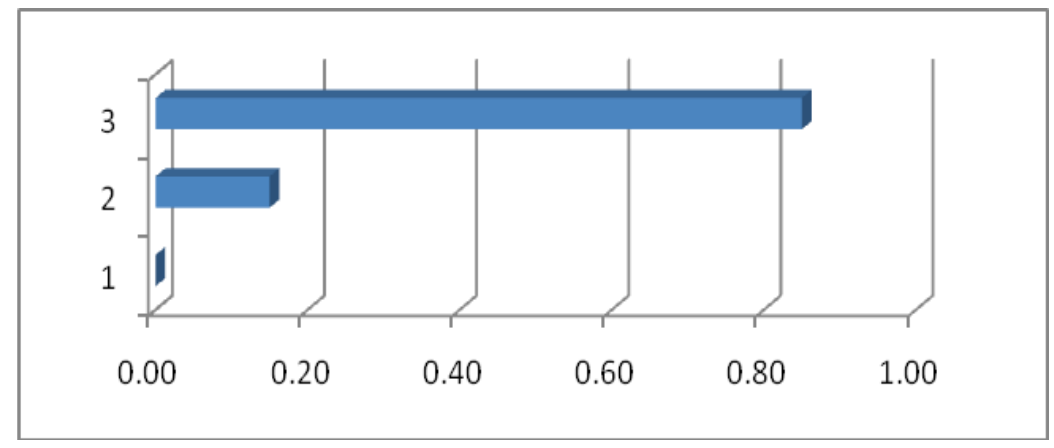

Realizando el proyecto he aprendido cosas útiles

No obstante, cabe comentar que en ciertas preguntas hemos obtenido resultados no concluyentes o resultados que muestran mayor dispersión y que conviene analizar detalladamente para tomar las acciones oportunas de rediseño, reorientación y mejora de la webquest. Así:

- El 53\% de los participantes no está nada de acuerdo o poco de acuerdo con realizar la actividad en grupo. El resultado que obtenemos en esta pregunta refuerza nuestra idea inicial de desligarnos del planteamiento generalizado de la webquest colaborativa y apostar por una webquest individual. Nuestras dudas acerca de las dificultades que pueden surgir en la puesta en práctica del trabajo colaborativo en la enseñanza a distancia, también son percibidas por los estudiantes cuyas opiniones se encuentran claramente divididas entre una y otra opción. Este resultado debe concatenarse con la reflexión sobre el distinto perfil que presenta el estudiante de la UNED respecto al de la universidad presencial.

- El 53\% de los participantes considera que la realización de esta actividad ha mejorado sus destrezas en el uso de la informática. Puede concluirse que una gran parte de los estudiantes universitarios ya tienen adquiridas las competencias informáticas que se exigen en el mundo de hoy.

- El 53\% de los participantes considera que la realización de esta actividad permite relacionar todos los temas del programa. El análisis de este resultado pone de manifiesto que los alumnos no perciben que la webquest cubra todos los aspectos de la asignatura, lo que nos lleva a concluir que para conseguir este objetivo, es necesario proponer de modo complementario otro tipo de técnicas o metodologías a desarrollar junto con ésta. Es una idea que ha de ser considerada en futuras experiencias.

- El $45 \%$ de los participantes considera que les ha supuesto mucho esfuerzo participar en el proyecto. Teniendo en cuenta que el estudiante de la UNED tiene su tiempo muy limitado al compatibilizar su formación con otras ocupaciones laborales o familiares, y que la preparación del contenido de las asignaturas para realizar correctamente las pruebas presenciales ya constituye de por sí un esfuerzo grande, 
este resultado es el esperado.

- El 58\% de los encuestados opina que la realización de esta actividad requiere más tiempo del previsto por el equipo docente. Este dato hace necesario revisar las horas estimadas para la realización de la webquest en ediciones futuras.

La dispersión encontrada en el análisis de las anteriores preguntas ha servido para que el equipo docente mejore algunos puntos propuestos en las tareas.

\section{CONCLUSIONES}

Los resultados obtenidos de la aplicación de la webquest a la asignatura Contabilidad de Empresas Turísticas han sido positivos. Los estudiantes que han participado en el proyecto manifiestan, en sus repuestas al cuestionario de satisfacción, que la actividad les ha servido para alcanzar los objetivos marcados y para dominar los conceptos básicos de la materia. Sus calificaciones finales han sido superiores a la media que obtuvieron los alumnos presentados a la prueba presencial. La evaluación de la experiencia pone de manifiesto el potencial que tiene esta estrategia de aprendizaje por descubrimiento para su formación. La experiencia les había resultado motivadora, e incluso los que rechazaban la asignatura en un principio por ser difícil de entender y compleja, remarcaban después que "hasta les resultaba interesante y atractiva".

En la vertiente de las competencias genéricas, el aprendizaje por descubrimiento les ha permitido desarrollar las capacidades de trabajo autónomo, análisis y síntesis, comunicación oral, y creatividad, además del ejercicio de las habilidades relacionadas con el uso de herramientas informáticas.

Los profesores que hemos participado en el proyecto también valoramos muy positivamente el trabajo realizado, y consideramos que ha sido una oportunidad para la implicación activa en la adaptación de la metodología al EEES. Pero es justo destacar, al mismo tiempo, el mayor esfuerzo en tiempo y dedicación que exige la puesta en práctica de estas metodologías respecto a las consideradas "tradicionales".

\section{BIBLIOGRAFÍA}

ADELL, J. (2004). Internet en el aula: las WebQuest. Edutec, Revista Electrónica de Tecnología Educativa, 17. Disponible en: http://www.uib.es/depart/gte/edutece/revelec17/adell_16a.htm [Fecha de consulta: 03/03/2010]

BARBA, C. (2002). La investigación en Internet con las WebQuest. Comunicación y Pedagogía, 185, 62-66. Reeditado en BARBA, C. (2004). La investigación en Internet con las WebQuest". Quaderns Digitals.Net. Número especial monográfico sobre WebQuest. 
http://www.quadernsdigitals.net/index.php?accionMenu=hemeroteca.VisualizaArticulolU.vi sualiza\&articulo_id=7365. [Fecha de consulta: 10/04/2009]

BERNABÉ, I; ADELL, J. (2006). El modelo WebQuest como estrategia de desarrollo de competencias genéricas en el EEES. Comunicación presentada en EDUTEC 2006. Disponible en: http://elbonia.cent.uji.es/jordi/wp-content/uploads/docs/iolanda-bernabe-munoz.pdf [Fecha de consulta: 12/05/2010]

BERNABÉ, I. (2007). WebQuests en el Espacio Europeo de Educación Superior: Un estudio de formación-acción. I Congreso Internacional Escuela y TIC. IV Forum Novadors. Más allá del Software Libre. Disponible en: http://www.dgde.ua.es/congresotic/public_doc/pdf/23295.pdf [Fecha de consulta: $17 / 11 / 2008]$

CONDE, A. y POZUELO, F. (2007). Las plantillas de evaluación (rúbrica) como instrumento para la evaluación. Un estudio de caso en el marco de la reforma de la enseñanza universitaria en el EEES. Investigación en la Escuela, 63, 77-90.

DEGROSSI, M.; CARNEVALI, S. (2009). Webquest y Edublog: Experiencia en la Enseñanza Universitaria de Toxicología de Alimentos. Ried, Revista Iberoamericana de Educación a Distancia, 12 (2), 211-228.

DODGE, B. (1999). WebQuest taskonomy: A taxonomy of tasks. Disponible en: http://Webquest.sdsu.edu/taskonomy.htmt [Fecha de consulta: 01/06/2000]

DODGE, B. (2001a). Five Rules for Writing a Great WebQuest. Learning \& Leading with Technology, 28 (8), 6-9.

DODGE, B. (2001b). A Rubric for Evaluating Webquest. Disponible en: http://edweb.sdsu.edu/webquest/webquestrubric.html [Fecha de consulta: 3/09/2003]

FERNÁNDEZ AEDO, R.; CARBALLOS, E. (2008). Un modelo de autoaprendizaje con integración de las TIC y los métodos de gestión del conocimiento. Ried, Revista Iberoamericana de Educación a Distancia, 11 (2), 137-149.

LARA, S. (2007). Eficacia de la WebQuest como una metodología acorde con el nuevo EEES: una experiencia univarsitaria. Revista de ciencias de la Educación, 210, 135-152.

MARCH, T. (2000). Las 3 Rs de las búsquedas en la red. Mantengámoslas Reales, Ricas y Relevantes.

Disponible

en:

[http://www.xtec.es/ jrosell3/webquest/fitxers/tommarch_las3rs.pdf] [Fecha de consulta: 3/06/2008]

MARCH, T. (2004). The learning power of WebQuest. Educational Leadership, 61 (4), 42-47.

PALACIO, A. (2009). Las webquest como estrategias metodológicas ante los retos de la convergencia europea de educación superior. Píxel Bit, Revistas de Medios y Comunicación, $34,235-249$

RODERA, A. M. (2008). Catalogación y valoración de las webquests desde el área de educación física y el tratamiento de temáticas transversales. Edutec, Revista Electrónica de Tecnología Educativa, 27, Noviembre. Disponible en: http://edutec.rediris.es/Revelec2/revelec27/articulos_n27_PDF/Edutec-E_Rodera_n27.pdf 
[Fecha de consulta: 05/04/2010]

Para citar este artículo:

DEL CAMPO, P. \& PARTE, L. (2011) .Edutec-e, Revista Electrónica de Tecnología Educativa, 38. Recuperado el dd/mm/aa de

http://edutec.rediris.es/Revelec2/Revelec38/webquest_estrategia_metodologica_ensenanz a_universitaria_contabilidad_empresas_turisticas.html 